\title{
The influence of hands posture on mental rotation of hands and feet
}

\author{
Silvio Ionta · Alissa D. Fourkas · Mirta Fiorio · \\ Salvatore M. Aglioti
}

Received: 3 October 2006/ Accepted: 8 May 2007/Published online: 21 July 2007

(C) Springer-Verlag 2007

\begin{abstract}
Behavioural and functional neuroanatomy studies demonstrate that mental rotation of body parts is carried out through a sort of inner motor simulation. Here we examined whether changes of hands posture influence the mental rotation of hands and feet. Twenty healthy subjects were asked to verbally judge the laterality of hands and feet pictures in two different postural conditions. In one condition, subjects kept hands on their knees in anatomical position; in the other, their hands were kept in an unusual posture with intertwined fingers, behind the back. Results show that mental rotation of hands but not of feet was influenced by changes in hands posture. Indeed, while mental rotation of hands was faster in the front than in the back hands position, no similar effect was found when
\end{abstract}

S. Ionta $(\bowtie)$

Department of Clinical Sciences and Bioimaging, Institute of Advanced Biomedical Technologies, University of Chieti-Pescara "G. D'Annunzio", via dei Vestini 33, 66013 Chieti Scalo, Italy

e-mail: silvio.ionta@uniroma1.it

\author{
A. D. Fourkas · S. M. Aglioti ( $\square)$ \\ Department of Psychology, \\ University of Rome "La Sapienza", \\ via dei Marsi 78, 00185 Rome, Italy \\ e-mail: salvatoremaria.aglioti@uniroma1.it
}

M. Fiorio

Department of Neurological and Vision Sciences,

Section of Rehabilitative Neurology, University of Verona,

Strada le Grazie 8, 37134 Verona, Italy

\section{S. M. Aglioti}

Neuropsychology Research Centre,

IRCCS "Fondazione Santa Lucia",

via Ardeatina 306, 00179 Rome, Italy mentally rotating feet. Thus, sensory-motor and postural information coming from the body may influence mental rotation of body parts according to specific, somatotopic rules.

Keywords Mental rotation - Laterality task . Motor imagery $\cdot$ Posture $\cdot$ Body schema

\section{Introduction}

Mental rotation tasks imply imagination of how an object would look if rotated away from the orientation in which it actually appears (Thayer et al. 2001). Different types of stimuli, such as three-dimensional figures (Cohen et al. 1996; Shepard and Metzler 1971), alphanumeric characters (Corballis and Sergent 1989) and body parts (Parsons 1987a, 1994) have been used. The mental rotation of body parts is a cognitive task in which subjects imagine moving a given body part from its actual posture to that of the same observed or imagined body part. This process engages an anatomically interconnected system implicated in the integration of sensory information with motor actions. Mental motor rotation seems to require the integrity of specific cortical-subcortical motor systems involved in motor planning and execution, such as motor and premotor areas and basal ganglia (Michelon et al. 2006; Bonda et al. 1995; Ganis et al. 2000; Kosslyn et al. 1998; Parsons et al. 1995; Alivisatos and Petrides 1997). Mental motor rotation of body parts in space shares the same temporal and kinematic properties with actual body transformations in space (Shepard and Metzler 1971; Decety et al. 1989, 1991, 1994; Parsons 1994; Parsons et al. 1998; Porro et al. 1996; Gerardin et al. 2000). Indeed, longer mental rotation times are usually observed for stimuli orientations corresponding 
to body part positions that would be actually difficult to maintain (Parsons 1994; Thayer et al. 2001; Petit et al. 2003; Fiorio et al. 2006).

A behavioral task typically used for assessing mental rotation of a body part is the so-called laterality judgment, in which subjects are requested to report the laterality (left or right) of depicted body parts, such as hands or feet, presented in different views and angular orientations. Hands laterality judgement depends on a specific sensorymotor mental simulation (Parsons et al. 1998) and it is influenced by the spatial origin from which one spontaneously imagines a transformation of one's own body (Parsons 1994).

Variables like the point of view from where the stimulus is seen, the stimulus laterality, and the complexity of the movements to be mentally executed may influence mental rotation of body parts (Parsons 1994). Relevant to the present study is the notion that mental motor rotation of body parts seems to be performed through the observer's inner simulation of actual rotational movement necessary to align the stimulus with a canonical orientation and to be influenced by proprioceptive information regarding current limb position (Parsons 1994). Thus, in principle, different postures and actual biomechanical bodily constraints of the subject performing the task can influence mental motor rotation of body parts. In keeping with this notion is the result that actual hand posture, such as holding the hands on the lap or in the back, influenced the ability of normal subjects to perform a hand mental rotation task (Sirigu and Duhamel 2001).

We explored whether the body-posture effect is specific for the body part that is to be mentally rotated by using a mental rotation task of hands and feet and varying the subject's hands posture. The main aims of the study were to assess whether the posture of the hands influences performance, and if any effect of hands posture is also found when other body parts, such as feet, are mentally rotated. Moreover, we investigated whether any postural effect on mental rotation was pertinent to the orientation of the stimuli to be mentally rotated or to the stimulus view (e.g. palmar or dorsal).

\section{Methods}

\section{Participants}

Twenty healthy subjects (7 female) aged 20-35 years $(M=26.4$ years, $\mathrm{SD}=3.9)$ participated in the experiment. All subjects were right-handed according to a standard handedness inventory (Briggs and Nebes 1975). The protocol was approved by the local ethics committee and the research was conducted in accordance with the 1964
Declaration of Helsinki. Written informed consent was obtained prior to participation.

\section{Materials}

The experimental stimuli consisted of naturalistic pictures of hands or feet, presented one at a time on a computer screen (Fiorio et al. 2006). Left hands and feet were mirror images of the original right ones. Stimuli could be presented in four different views (dorsum, little finger/toe side, thumb/big toe side, palm/plantar) and oriented in one of five clockwise orientations from the upright $\left(60^{\circ}, 120^{\circ}\right.$, $\left.180^{\circ}, 240^{\circ}, 300^{\circ}\right)$. The upright orientation was defined as fingers/toes pointing upwards $\left(0^{\circ}\right)$.

\section{Procedure}

Subjects were seated in front of a computer screen (Apple ColorSync), positioned $60 \mathrm{~cm}$ distant from the subject's eyes. Stimuli presentation was controlled with E-Prime (Psychology Software Tools Inc., Pittsburgh, USA). After the presentation of each stimulus, subjects verbally judged the laterality (left or right) of hands or feet, as quickly and accurately as possible. The stimulus remained visible on the screen until the response was given. Response time was automatically recorded by a microphone connected to the computer. Response accuracy was manually recorded by an experimenter.

Subjects were tested in one experimental session consisting of four blocks. Two blocks contained 96 pictures of hands and other two blocks contained 96 pictures of feet. Each of the two hands, and feet, stimuli type blocks was characterized by a specific upper limb posture physically maintained by the subjects, as depicted in Fig. 1. In one hand block, subjects positioned their hands on their knees (Front condition), while in the other block they held their hands behind their back with the fingers intertwined (Back
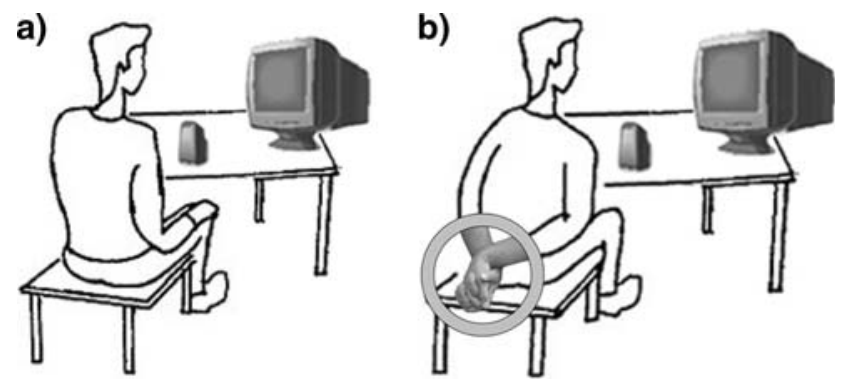

Fig. 1 Schematic representation (modified from Sirigu and Duhamel 2001) of the two hands postures and how the back posture differs from that used previously. a Our front hand posture is identical to that used by Sirigu and Duhamel. b The circle frames the photo which illustrates the back hand posture which subjects assumed in the current study. In order to mentally move the hand into the position of the stimulus, the fingers must be un-entwined and hands uncrossed 
condition); the same postural manipulation was used for the blocks of feet stimuli. The order of the four blocks was counterbalanced across subjects.

\section{Data analysis}

We measured accuracy and response time (RT). Accuracy was defined as the number of correct responses (in percentage) in relation to the original total number of responses per variable prior to response time filtering. Response time was defined as the time between the stimulus onset and the subject's verbal response. Previous studies using similar laterality tasks reported that response times to hand stimuli range between 500 and $3,500 \mathrm{~ms}$ (Sekiyama 1982; Kosslyn et al. 1998, 2001; Cooper and Shepard 1975; Parsons 1987b, 1994; Wohlschlager and Wohlschlager 1998). Therefore, trials in which response times were faster than $500 \mathrm{~ms}$ or slower than $3,500 \mathrm{~ms}$ were excluded from the data analyses (total loss, $5 \%$ of trials); then trials with allowable response times but incorrect responses were excluded (total loss, $7 \%$ of trials) from the analysis of response time.

Response time and accuracy were analysed by means of four separate repeated measures analysis of variance (ANOVA). Two series of analyses included body part (hands, feet), hands posture (front, back), stimulus laterality (left, right) and stimulus orientation $\left(0^{\circ}, 60^{\circ}, 120^{\circ}\right.$, $180^{\circ}, 240^{\circ}, 300^{\circ}$ ) as main factors. The other two included body part, hands posture, stimulus laterality and stimulus view (dorsum, little toe/finger, big toe/thumb, plantar/ palm) as main factors. Epsilon corrections are reported, where necessary, as Greenhouse-Geisser $(\hat{e})$ when HuyhtFeldt $(\tilde{e})$ epsilon was less than 0.75 . Post-hoc analyses were carried out using simple main effects (Bonferroni corrected) and the Newman-Keuls test $(P<0.05)$. The measure of the strength of association is reported as partial eta squared $\left(\eta_{\mathrm{p}}^{2}\right)$, the proportion of variance in the dependent variable attributable to the effect, as recommended by Tabachnick and Fidell (1989).

Finally, in order to tease apart different components of the mental rotation process, we performed regression lines on RT for the different stimulus orientations and calculated intercepts and slopes. Intercept is believed to reflect the contribution on non-rotational processes, while slope reflects the average change as orientation increases.

\section{Results}

\section{Orientation}

Response times and accuracy at each orientation for both hand postures in the hand and feet laterality tasks are graphically reported in Fig. 2. The response time ANOVA showed a significant main effect of Hands posture $\left[F(1,19)=6.873, P=0.017, \eta_{\mathrm{p}}^{2}=0.27\right]$, stimulus laterality $\left[F(1,19)=38.132, P<0.000, \eta_{\mathrm{p}}^{2}=0.67\right]$, stimulus orientation $\left[F(5,95)=17.545, P<0.000\right.$ with $\hat{e}=0.37, \eta_{\mathrm{p}}^{2}=$ $0.48]$, and a body part by hands posture interaction $\left[F(1,19)=7.052, P=0.016, \eta_{\mathrm{p}}^{2}=0.27\right]$. The hands posture effect was accounted for by the slower performance in the back condition $(1,257 \mathrm{~ms})$ with respect to the front condition $(1,188 \mathrm{~ms})$. The stimulus laterality effect was accounted for by the slower performance for left stimuli $(1,280 \mathrm{~ms})$ with respect to right stimuli $(1,165 \mathrm{~ms})$. The stimulus orientation effect was accounted for by the slowest performance at $180^{\circ}(1,358 \mathrm{~ms})$ compared to all other orientations; the three fastest orientations $\left[0^{\circ}(1,145 \mathrm{~ms}), 60^{\circ}(1,167 \mathrm{~ms})\right.$ and $\left.300^{\circ}(1,176 \mathrm{~ms})\right] \mathrm{did}$ not differ from one another. The remaining intermediate orientations $\left[120^{\circ}(1,256 \mathrm{~ms}), 240^{\circ}(1,234 \mathrm{~ms})\right]$ differed from all orientations except each other.

The body part by hands posture interaction, shown in Fig. 3 (left side), was accounted for by the hand stimuli $[F(1,19)=13.624, P=0.002]$. Specifically, the performance for hands stimuli judged with the hands in back $(1,311 \mathrm{~ms})$ was slower than to the same stimuli judged with the hands in front $(1,162 \mathrm{~ms})$. Critically, there is no difference $(P=0.789)$ between the two postural conditions for the feet stimuli (back 1,203 ms; front 1,214 ms); there were no differences between body parts according to hands posture (front, $P=0.280$; back, $P=0.163$ ). Additionally, we computed the slopes and intercepts of the four components (Fig. 3 right side). A linear function from $0^{\circ}$ to $180^{\circ}$ was tested after combining data equidistant from $180^{\circ}$ (i.e., $60^{\circ}$ with $300^{\circ}$, and $120^{\circ}$ with $240^{\circ}$ ). Slope reflects the average change associated with rotating an object an additional degree and the intercept reflects contributions of non-rotational processes. Hand stimuli in the front posture yielded a less steep slope $\left(y=62.816 x+1014, R^{2}=0.83\right)$. The remaining three components had similar slopes (hand stimuli in the back, $y=77.034 x+1126, R^{2}=0.93$; feet stimuli in the front, $y=73.899 x+1032, R^{2}=0.99$; feet stimuli in the back, $\left.y=71.317 x+1034, R^{2}=0.89\right)$. This seems to reflect that $180^{\circ}$ did not require as large of a change in response time for hand stimuli in the front posture, as can be discerned from Fig. 2. Hand stimuli in the back posture had the highest intercept indicating an additional non-rotational process. It can be further noted in Fig. 2 that responses at $0^{\circ}$ are noticeably longer in this condition (Fig. 4).

The ANOVA for accuracy showed a significant body part by orientation interaction $[F(5,95)=2.852, P=0.023$ with $\left.\tilde{e}=0.91, \eta_{\mathrm{p}}^{2}=0.13\right]$, and a trend toward significance for hands posture $\left[F(1,19)=3.703, P=0.069, \eta_{\mathrm{p}}^{2}=0.16\right]$. Analysis of the interaction failed to find simple main 

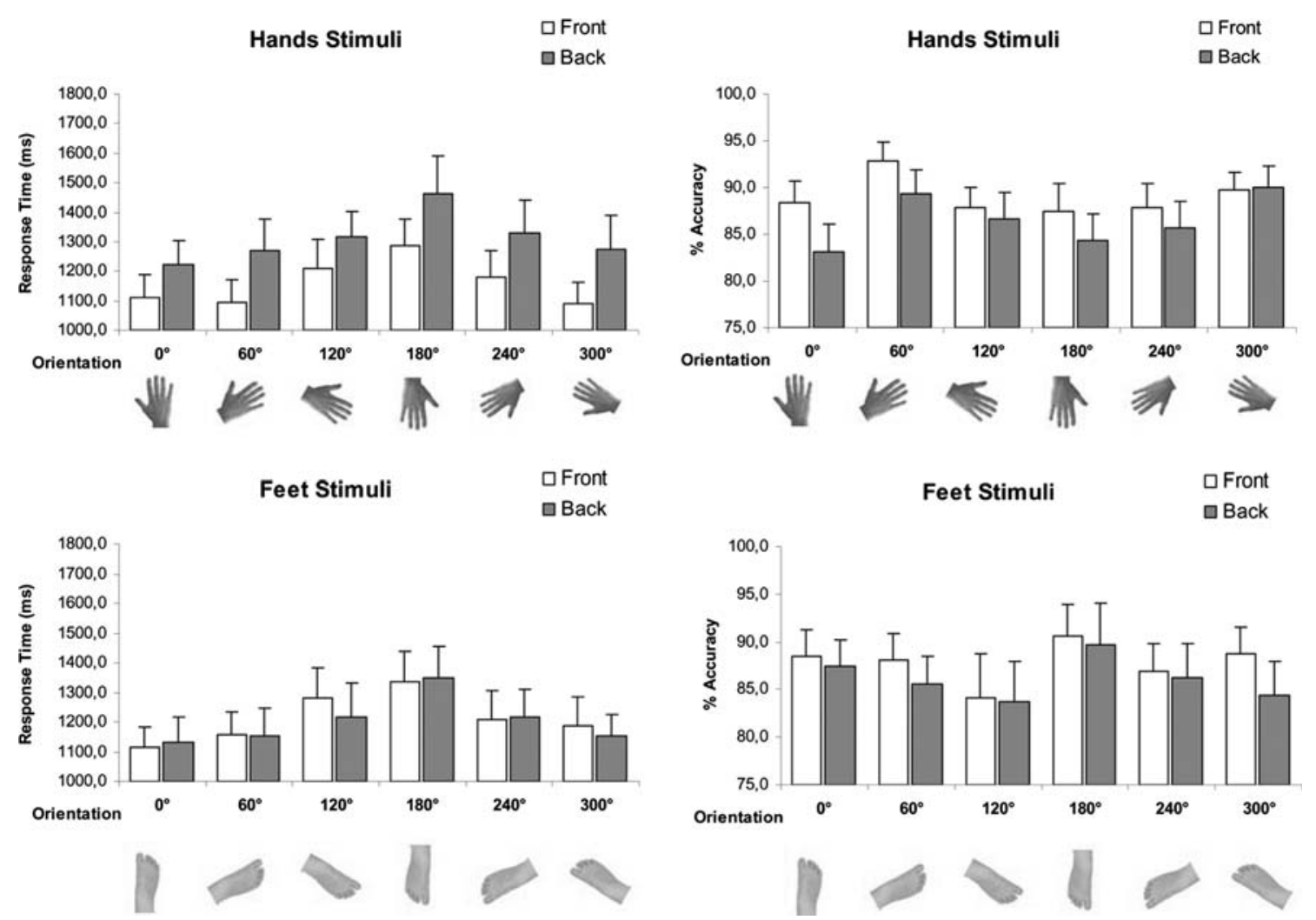

Fig. 2 Mean response times (left part of the figure) and accuracy (right part of the figure) at the different hands (top) and feet (bottom) stimuli orientations in front and back postural conditions. Error bars depicts the standard error of the mean

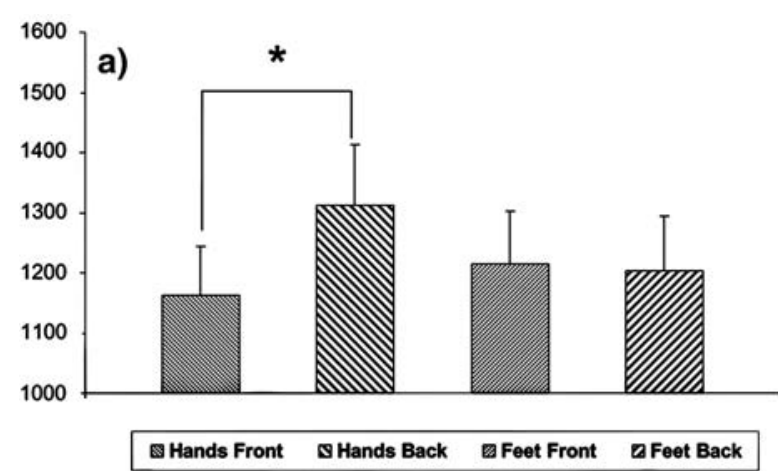

Fig. 3 Posture effect (left part of the figure) and intercepts (right part of the figure). a Mean response time for hand (left) and feet (right) judged in the two different postural conditions. Error bars depicts the

effects that survived Bonferroni correction $(P \leq 0.006)$, the closest trend being for accuracy to vary with orientation in the hand stimuli $(P=0.016)$. The trend suggested less accurate performance in the back condition $(86 \%)$ with respect to the front condition $(88 \%)$.

View

Response times and accuracy for both hand postures at the different stimuli views in the hand and feet laterality task

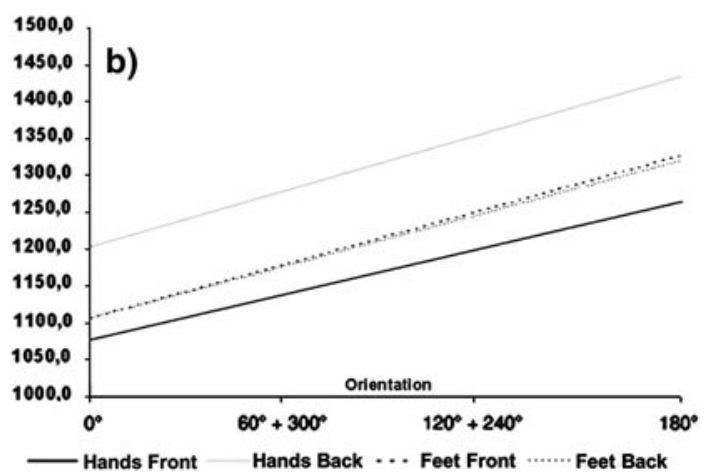

standard error of the mean; b Least square regression lines; intercepts indicating the contribution of non-rotational processes

are graphically reported in Fig. 3. The ANOVA on response time showed a main effect of stimulus view $\left[F(3,57)=29.624, P<0.000\right.$ with $\left.\tilde{e}=1.0, \eta_{\mathrm{p}}^{2}=0.61\right]$ and a body part by view interaction $[F(3,57)=8.549$, $P<0.000$ with $\left.\tilde{e}=0.76, \eta_{\mathrm{p}}^{2}=0.31\right]$, as well as the main effect of hands posture $\left[F(1,19)=8.745, P=0.008, \eta_{\mathrm{p}}^{2}=\right.$ $0.32]$, stimulus laterality $[F(1,19)=33.367, P<0.000$, $\left.\eta_{\mathrm{p}}^{2}=0.64\right]$, and the body part by hands posture interaction $\left[F(1,19)=7.830, \quad P<0.011, \quad \eta_{\mathrm{p}}^{2}=0.29\right]$. The stimulus view effect revealed that all views differed significantly 

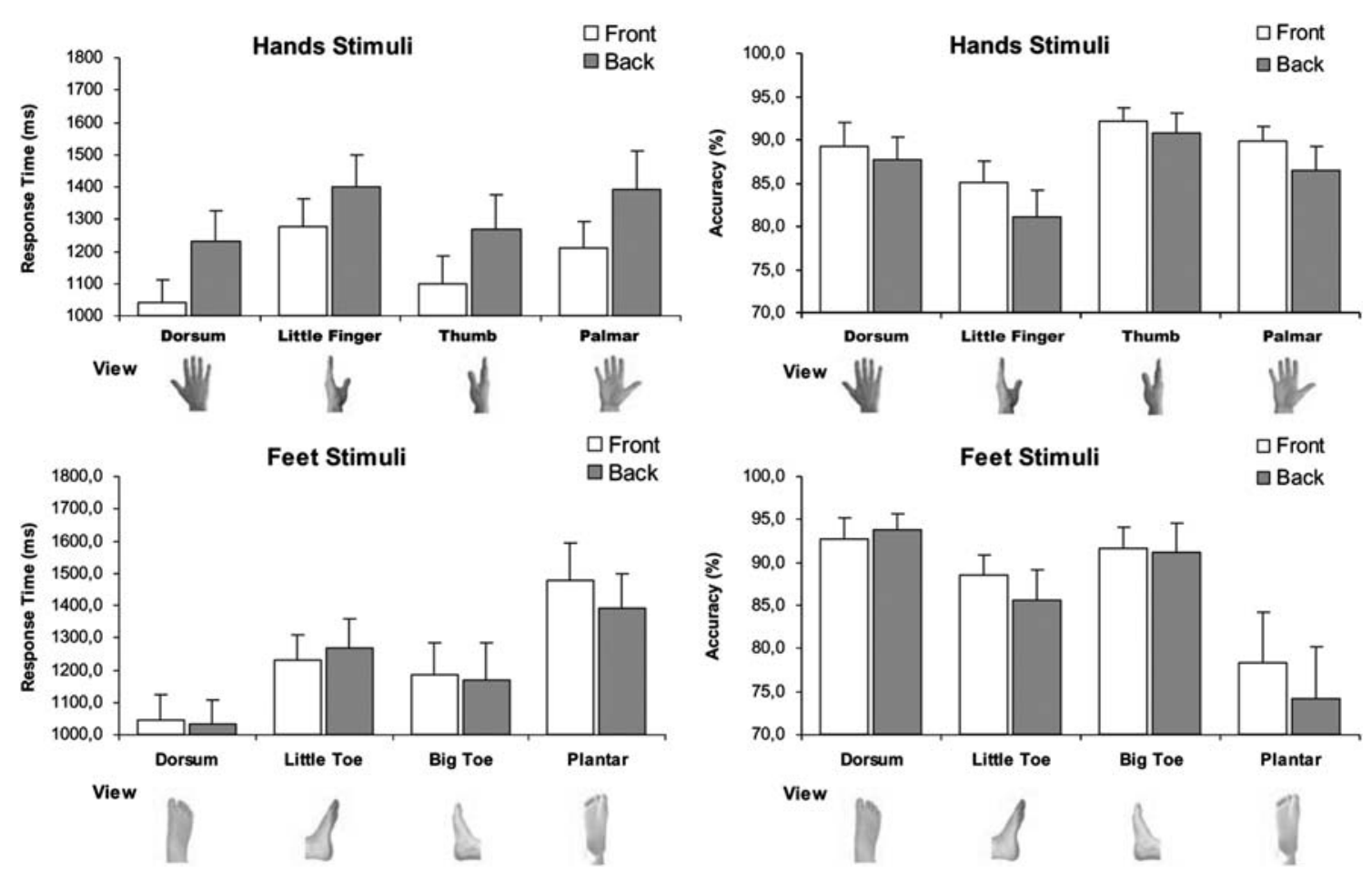

Fig. 4 Mean response times (left part of the figure) and accuracy (right part of the figure) at the different hand (up) and feet (down) stimuli views in front and back postural conditions. Error bars depict the standard error of the mean

from one another; the fastest responses were for the dorsum $(1,087 \mathrm{~ms})$ and slowest for the palm/plantar $(1,368 \mathrm{~ms})$ view, while the thumb/big toe $(1,180 \mathrm{~ms})$ and little finger/ toe $(1,294 \mathrm{~ms})$ views fell in between. The interaction between body part and view was explained by response times varying with the view of the body part being judged. When judging hand stimuli $[F(3,57)=16.695, P<0.000$ with $\tilde{e}=0.99]$, responses to the dorsum $(1,135 \mathrm{~ms})$ and thumb $(1,182 \mathrm{~ms})$ views were faster than responses to the palm $(1,340 \mathrm{~ms})$ and little finger $(1,300 \mathrm{~ms})$ views. When judging feet stimuli $[F(3,57)=23.532, P<0.000$ with $\tilde{e}=0.95]$, responses to the dorsum $(1,040 \mathrm{~ms})$ were significantly faster, and responses to the plantar view $(1,434 \mathrm{~ms})$ significantly slower, than to all other views; responses to the big toe $(1,178 \mathrm{~ms})$ and little toe $(1,249 \mathrm{~ms})$ did not differ from each other. None of the four views, however, differed according to body part (dorsum, $P=0.053$; little finger/toe, $P=0.174$; thumb/big toe, $P=0.949 ;$ palm/plantar, $P=0.094$ ).

The analysis of accuracy showed a significant main effect of stimulus view $[F(3,57)=10.770, P<0.000$ with $\left.\hat{e}=0.59, \eta_{\mathrm{p}}^{2}=0.36\right]$, an interaction between body part and view $\left[F(3,57)=7.513, P=0.003\right.$ with $\hat{e}=0.57, \quad \eta_{\mathrm{p}}^{2}=$ $0.28]$, and the trend for hands posture $[F(1,19)=3.703$, $\left.P=0.069, \eta_{\mathrm{p}}^{2}=0.16\right]$ noted above. Performance was significantly less accurate when rotating stimuli seen from the palm/plantar (82\%) and the little finger/toe (85\%) sides than from the dorsum $(91 \%)$ and the thumb/big toe $(91 \%)$ sides. The interaction revealed that accuracy varied according the view in different ways for hands and feet stimuli. Accuracy for the hands stimuli $[F(3,57)=7.158, P=0.001$, with $\tilde{e}=0.79$ ] was less accurate when the little finger $(83 \%)$ view was shown than when other views were shown (dorsum, $88 \%$; thumb, 91\%; palm, $88 \%$ ). In contrast, accuracy for the feet stimuli $[F(3,57)=9.656, P=0.003$ with $\hat{e}=0.42]$ was lowest when the plantar $(76 \%)$ view was shown (dorsum, 93\%; little toe, $87 \%$; big toe, $91 \%$ ). Body part did not differ within each view when Bonferroni corrected (dorsum, $P=0.044$; little finger/toe, $P=0.205$; thumb/big toe, $P=1.0$; palm/plantar, $P=0.029$ ).

\section{Discussion}

The ability to perform mental rotation of body parts is strictly linked to the concept of "body schema", a term that alludes to the complex of sensations, perception, memories and ideas about one's own and others' anatomy (Berlucchi and Aglioti 1997). This mental construct is a plastic representation of the body and its movements, which allows people to act in the environment and it is influenced by factors such as pain (Schwoebel et al. 2001) and posture 
(Sirigu et al. 1995; Dominey et al. 1995; Sekiyama 1982; Parsons 1994). The process of mentally representing the body is likely to rely upon the integration of information coming from different modalities, such as proprioceptive, somatosensory, visual and vestibular. Merging these different types of information leads to the general multisensory experience of our body and its position in space (Berlucchi and Aglioti 1997).

The present study shows that the current posture of the hands can selectively influence a restricted portion of the body schema, by having an effect on the mental rotation of the very same body part. More specifically, holding the hands in the back had an influence on subjects' performance, in that it impaired response time during mental rotation of hands. In the feet mental rotation task, we did not observe any hands posture related modulation of reaction time. This suggests that subject's hands posture during laterality judgments does not play any major role when the task regards body parts other than the hands, such as feet.

It has been demonstrated that during mental rotation of body parts subjects simulate movements of their body segments so as to match that of the observed stimulus (Parsons 1994). Thus, the position of subject's own body plays an important role in the ability to mentally represent body parts in space. The effect of body posture has been shown by Parsons (1994) in the context of a handedness decision task in which RTs were longer when subjects kept their hands outstretched forward than resting on the lap.

Sirigu and Duhamel (2001) tested healthy subjects in a body part imagery task performed according to an egocentric, first-person imagery strategy. A clear impairment of performance was observed when subjects kept the hands behind their back with respect to when they kept hands on the lap (Sirigu and Duhamel 2001). The present study extends previous knowledge by suggesting that the posture effect is specific insofar as the abnormal posture of hands influences the mental rotation of hands but not of feet.

In order to build an accurate and up-to-date representation of our body parts in space mainly two types of information, visual and proprioceptive, are integrated. Information coming from the visual and proprioceptive modalities contributes differentially to the computation of body representation. While vision may be essential for representing the body in space, proprioception may be crucial for determining the relationship between different body segments. Both modalities, however, contribute to building and maintaining the representation concerning the position of a given body part, that is fundamental to planning subsequent goal-directed actions. Since visual information of the hands was prevented in our task, the impairment found is likely to be due to a short-term perturbation of the proprioceptive system, caused by the hand posture. This is in keeping with data showing that under appropriate circumstances, proprioceptive inflow may represent the dominant sensory input to the online representation of the body in space (Shenton et al. 2004). Different lines of evidence converge to indicate a tight link between body schema alterations and impairments in hand laterality tasks. It has been demonstrated, for example, that patients suffering from chronic arm pain (Schwoebel et al. 2001) or from complex regional pain syndrome (Moseley 2004) show a specific impairment in the mental rotation of the body part affected by pain. Moreover, a study in upper limb amputees showed that judging the laterality of body parts was more difficult in patients with amputation of dominant than non dominant limb (Nico et al. 2004). The close relationship between actual sensorimotor disturbance and mental rotation deficits has been suggested also by a study in patients with focal hand dystonia, a neurological disease characterised by sustained muscular contractions localized to the dominant hand (Fiorio et al. 2006). These patients were slower than control subjects in mentally rotating pictures of a hand (the affected body part) but not of another body segment, such as the foot.

The findings we obtained in regard to the stimulus view complement those of Parsons (1987b), in that responses to the palm view were slower than to the dorsum view. However, previous findings have been here extended by showing that the little finger view leads to the slowest (together with palm) and least accurate performances for hand stimuli. In the case of the feet stimuli, the slowest and least accurate performances were for the plantar view. These results can be seen as an evidence of the fact that the little finger view (for the hands) and the plantar view (for the feet) were the most biomechanically difficult mental spatial transformations that subjects had to perform in order to rotate their own hand and foot into the stimulus position. Additionally, the performance impairments may have been influenced by the fact that the little finger and the plantar views are the less common views of one's own hand and foot that a person would normally see and process. This reduced amount of relevant visual information stored in memory would presumably force greater reliance on proprioceptive information.

Another interesting result of our study is the strong effect of stimulus laterality on reaction times. In particular, right stimuli (hand and foot) were processed faster than left stimuli. A similar laterality effect has been also reported by Parsons (1987b) and Gentilucci et al. (1998), who noticed that right-handed subjects responded faster to right than to left hands and feet (Parsons 1987b; Gentilucci et al. 1998). According to Gentilucci et al., this may be due to the greater lateralization of hand motor skills in right-handed people, or that the motor strategy of mental rotation is slowed on the less used non-dominant side. Combined with the evidence that less accurate and slower performances were found for 
more biomechanically difficult orientations and views, this suggests the use of a motor strategy of rotating ones' own body part into the position of the stimulus.

The novel result of the present research is the specificity of the relation between body posture and mental imagery of body parts. In particular, subject's hands postural variations determined significant differences in the hands task, but not in the feet task. This suggests that the actual posture of specific body parts plays a specific influence on the mental rotation of the same body districts. Our suggestion is that afferent information from the subject's body parts specifically influenced the mental rotation of the same body part in a bottom-up manner.

Acknowledgments This research was supported by Grants from the Ministero Istruzione Universita' e Ricerca and Fondo Investimenti Ricerca di Base, Italy both awarded to SMA. We would like to thank the anonymous reviewers for their helpful comments.

\section{References}

Alivisatos B, Petrides M, (1997) Functional activation of the human brain during mental rotation. Neuropsychologia 35(2):111-118

Berlucchi G, Aglioti SM, (1997) The body in the brain: neural bases of corporeal awareness. Trends Neurosci 20(12):560-4

Bonda E, Petrides M, Frey S, Evans A (1995). Neural correlates of mental transformation of the body-in-space. Proc Natl Acad Sci USA. 92:11180-11184

Briggs CG, Nebes RD (1975) Patterns of hand preference in a student population. Cortex 11:230-238

Cohen MS (1996) Changes in cortical activity during mental rotation: a mapping study using functional magnetic resonance imaging. Brain 119:89-119

Cooper LA, Shepard RN (1975) Mental transformations in the identification of left and right hands. J Exp Psychol Hum Percept Perform 104(1):48-56

Corballis MC, Sergent J (1989) Hemisferic specialization for mental rotation. Cortex 25(19):15-25

Decety J, Jeannerod M, Prablanc C (1989) The timing of mentally represented actions. Behav Brain Res 34:35-42

Decety J, Jeannerod M, Germain M, Pastene J (1991) Vegetative response during imagined movement is proportional to mental effort. Behav Brain Res 42:1-5

Decety J, Perani D, Jeannerod M, Bettinardi V, Tadary B, Woods RP, Mazziotta JC, Fazio F (1994) Mapping motor representations with positron emission tomography. Nature 371:600-602

Dominey P, Decety J, Broussolle E, Chazot G, Jeannerod M (1995) Motor imagery of a lateralized sequential task is asymmetrically slowed in hemi-Parkinson's patients. Neuropsychologia 33:727741

Fiorio M, Tinazzi M, Aglioti SM (2006) Selective impairment of hand mental rotation in patients with focal hand dystonia. Brain 129(Pt 1):47-54

Ganis G, Keenan JP, Kosslyn SM, Pascual-Leone A, (2000) Transcranical magnetic stimulation of primary motor cortex affects mental rotation. Cereb Cortex 10:175-180

Gentilucci M, Daprati E, Gangitano M, (1998) Right-handed and lefthanded have different representation of their own hand. Brain Res Cogn Brain Res 6(3):185-192
Gerardin E, Sirigu A, Lehericy S, Poline JB, Gaymard B, Marsault C, Agid Y, Le Bihan D (2000) Partially overlapping neural networks for real and imagined hand movements. Cereb Cortex 10(11):1093-1104

Kosslyn SM, Di Girolamo GJ, Thompson WL, Alpert NM (1998) Mental rotation of objects versus hands: neural mechanisms revealed by positron emission tomography. Psychophysiology 35(2):151-161

Kosslyn SM, Ganis G, Thompson WL (2001) Neural foundations of imagery. Nat Rev Neurosci 2(9):635-42

Michelon P, Vettel JM, Zacks JM (2006) Lateral somatotopic organization during imagined and prepared movements. J Neurophysiol 95(2):811-22

Moseley GL (2004) Why do people with complex regional pain syndrome take longer to recognize their affected hand? Neurology 62:2182-2186

Nico D, Daprati E, Rigal F, Parsons L, Sirigu A (2004) Left and right hand recognition in upper limb amputees. Brain 127:120-132

Parsons LM (1987a) Imagined spatial transformation of one's body. J Exp Psychol Gen 116(2):172-191

Parsons LM (1987b) Imagined spatial transformation of one's hands and feet. Cognit Psychol 19:178-241

Parsons LM (1994) Temporal and kinematic properties of motor behavior reflected in mentally simulated action. J Exp Psychol Hum Percept Perform 20(4):709-730

Parsons LM, Fox PT, Downs JH, Glass T, Hirsch TB, Martin CC, Jerabek PA, Lancaster JL (1995) Use of implicit motor imagery for visual shape discrimination as revealed by PET. Nature 375(6526):54-58

Parsons LM, Gabrieli JD, Phelps EA, Gazzaniga MS (1998) Cerebrally lateralized mental representations of hands shape and movement. J Neurosci 18(16):6593-6548

Petit LS, Pegna AJ, Mayer E, Hauert CA (2003) Representation of anatomical constraints in motor imagery: mental rotation of a body segment. Brain Cogn 51(1):95-101

Porro CA, Francescato MP, Cettolo V, Diamond ME, Baraldi P, Zuiani C, Bazzocchi M, di Prampero PE (1996) Primary motor and sensory cortex activation during motor performance and motor imagery: a functional magnetic resonance imaging study. J Neurosci 16(23):7688-7698

Schwoebel J, Friedman R, Duda N, Coslett HB (2001) Pain and the body schema: evidence for peripheral effects on mental representations of movement. Brain 124:2098-2104

Sekiyama K (1982) Kinesthetic aspects of mental representations in the identification of left and right hands. Percept Psychophys 32(2):89-95

Shenton JT, Schwoebel J, Coslett HB (2004) Mental motor imagery and the body schema: evidence for proprioceptive dominance. Neurosci Lett 370:19-24

Shepard NR, Metzler J (1971) Mental rotation of three-dimensional objects. Science 171:701-703

Sirigu A, Duhamel JR (2001) Motor and visual imagery as two complementary but neurally dissociable mental processes. J Cogn Neurosci 13(7):910-919

Sirigu A, Cohen L, Duhamel JR, Pillon B, Dubois B, Agid Y, PierrotDeseilligny C (1995) Congruent unilateral impairments for real and imagined hand movements. NeuroReport 6:997-1001

Tabachnick BG, Fidell LS (1989) Using multivariate statistics, 2nd edn. Harper and Row, New York

Thayer ZC, Johnson BW, Corballis MC, Hamm JP (2001) Perceptual and motor mechanisms for mental rotation of human hands. NeuroReport 16:3433-3437

Wohlschlager A, Wohlschlager A (1998) Mental and manual rotation. J Exp Psychol Hum Percept Perform 24(2):397-412 